$\mathbb{T}$ periodica polytechnica

\author{
Transportation Engineering \\ $3 8 / 1 ( 2 0 1 0 ) \longdiv { 2 9 } 3 5$ \\ doi: 10.3311/pp.tr.2010-1.06 \\ web: http://www.pp.bme.hu/tr \\ (c) Periodica Polytechnica 2010
}

RESEARCH ARTICLE

\section{Face dog clutch engagement at low mismatch speed}

\author{
Gergely Bóka / János Márialigeti / László Lovas / Balázs Trencséni \\ Received 2009-10-01
}

\begin{abstract}
Face dog clutches regain popularity in heavy duty AMT's. Though the reduction of the speed difference between the dogs is the key to a smooth gear shift, the excellent engaging capability is experienced to degrade at low mismatch speed and the outcome of the engagement process becomes uncertain. Using a simple mechanical model, that uncertainty is described and the probability of the successful engagement is given as a function of the initial mismatch speed. Results are validated through statistical evaluation of measurement data.
\end{abstract}

\section{Keywords}

heavy duty AMT · face dog clutch · mismatch speed · statistical evaluation

\section{Acknowledgement}

This work is connected to the scientific program of the "Development of quality-oriented and harmonized $R+D+I$ strategy and functional model at BME" project. This project is supported by the New Hungary Development Plan (Project ID: TÁMOP4.2.1/B-09/1/KMR-2010-0002).

\section{Gergely Bóka}

Department of Vehicle Parts and Drives, BME, H-1111 Budapest, Bertalan L. u. 2, Hungary

e-mail: boka@kge.bme.hu

\section{János Márialigeti}

László Lovas

Department of Vehicle Parts and Drives, BME, H-1111 Budapest, Bertalan L. u. 2, Hungary

\section{Balázs Trencséni}

Department of Automobiles, BME, H-1111 Budapest, Stoczek u. 6., Hungary

\section{Face dog clutches in modern commercial vehicle gearboxes}

Automated Mechanical Transmissions (AMT) are made up of an automated single disc dry clutch and a constant mesh gearbox with gear control shafts actuated electronically and not by the driver, see e.g. [1] They have the efficiency, life expectancy and load capacity of a manual gearbox and the comfort benefits of the automation of the gear shifting process. Consequently, AMT's are the state of art for transmissions of heavy duty commercial vehicles.

In order to reduce the time need for the synchronization, thereby further increase the comfort through quicker gear shifts, a multi-disk brake is installed on the gearbox countershaft to effectively reduce the countershaft speed at upshifts. On the other hand, increasing the gearbox input shaft speed is possible through partial clutch engagement, usually necessary at downshifts. Since those features enable the synchronization without synchromesh [2], synchromesh mechanisms [3] may be replaced with simpler and mechanically more robust face dog clutches [4], realizing major advantages compared to synchromesh: longer life expectancy, higher transmittable torque and cost benefits.

\section{Modeling the face contact}

\subsection{Model development}

During the engagement process of a face dog clutch (Fig. 11) it is usually the faces of the teeth that first come in contact. There is an impact between the tooth faces that changes the mismatch speed i.e. the speed difference between the sliding dog and the gear wheel from $\Delta \omega_{0}$ to $\Delta \omega_{1}$. Since generally $\left|\Delta \omega_{1}\right|>0$, the faces slip until the teeth of the sliding dog become able to enter the slots of the gear wheel. That is called the face friction phase, and the face friction torque transmitted by the dog clutch reduces the mismatch speed. As soon as further axial movement of the sliding dog is possible, the dog clutch engages with a mismatch speed of $\Delta \omega_{2}$ that rapidly becomes zero resulting in damped 


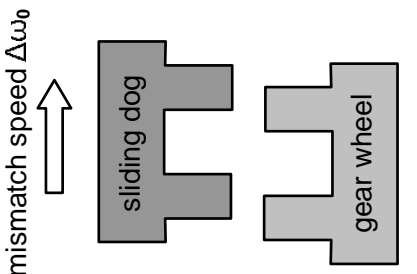

I. Free fly

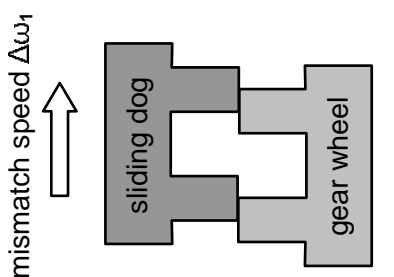

II. Beginning of face friction

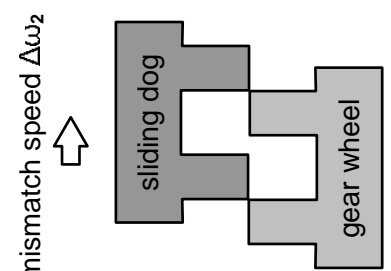

III. End of face friction

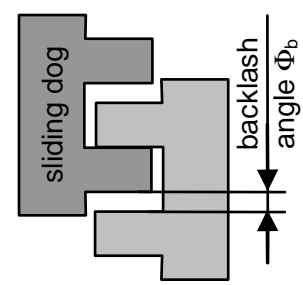

IV. Engagement

Fig. 1. Phases of the engagement process of a face dog clutch

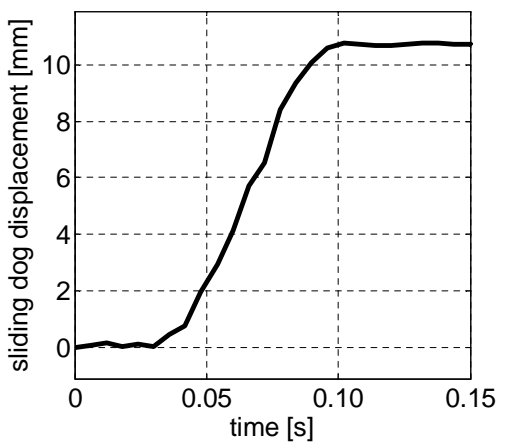

optimal engagement

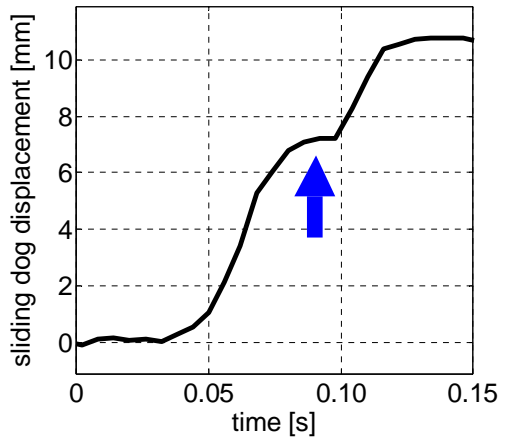

extended face contact

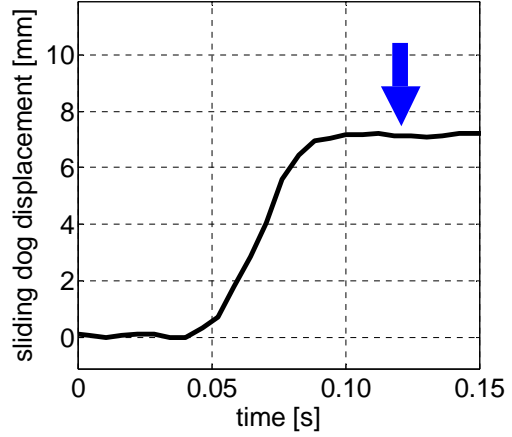

pernament face contact

Fig. 2. Variation in sliding dog displacement at low initial mismatch speed

torsional vibrations with peak value of

$$
T_{\max }=\left|\Delta \omega_{2}\right| \cdot \sqrt{\frac{k J_{1} J_{2}}{J_{1}+J_{2}}}
$$

where $k$ is the torsional stiffness of the dog clutch and the inertias $J_{1}$ and $J_{2}$ are the reduced inertias of the gear wheel and the sliding dog, respectively, considering gearbox and driveline ratios and masses. At a given gearbox design, the reduction of the mismatch speed is the only way to reduce torisonal vibrations and to ensure a smooth engagement.

During the analysis of the gear shifting process of an AMT with face dog clutch engagement at low mismatch speed there were some non-deterministic phenomena found. The measurement results in Fig. 2. clearly show the uncertainty of the outcome of the gear shift at $\Delta \omega_{0} \approx 3,2 \mathrm{rad} / \mathrm{s}$. Three cases are presented: seamless engagement, engagement with extended face contact and an unsuccessful engagement process ending up in permanent face contact. Note, that there is practically no difference between the initial mismatch speeds, major variation in the sliding dog axial movement is however experienced. In the following a simple model is built up to investigate the face contact of the dogs and to make the nature of that uncertainty clear.

Since not the complete engagement process but only the face contact has to be modeled, no detailed driveline model is needed at this point. Only one face dog clutch is considered, made up of a gear wheel and a sliding dog.

The driveline is considered in the $J_{1}$ and $J_{2}$ reduced inertias of the gear wheel and the sliding dog, respectively. Let $m_{2}$ denote the reduced mass of the sliding dog and the actuator attached to it. Accordingly, indices 1 and 2 for angular speeds and accelerations always refer to the gear wheel and to the sliding dog, respectively.

Let $\alpha$ denote the chamfer angle of the faces of the teeth, $r$ the mean radius of the contact zone, $z$ the number of the teeth (Fig. 3) and $\Phi_{b}$ the backlash angle (Fig. 1). The brake torque of the countershaft brake acting on the gear wheel is not considered and the $F_{a c t}$ axial force (Fig. 3) of the gearbox actuator acting on the sliding dog is assumed to be constant during the face contact.

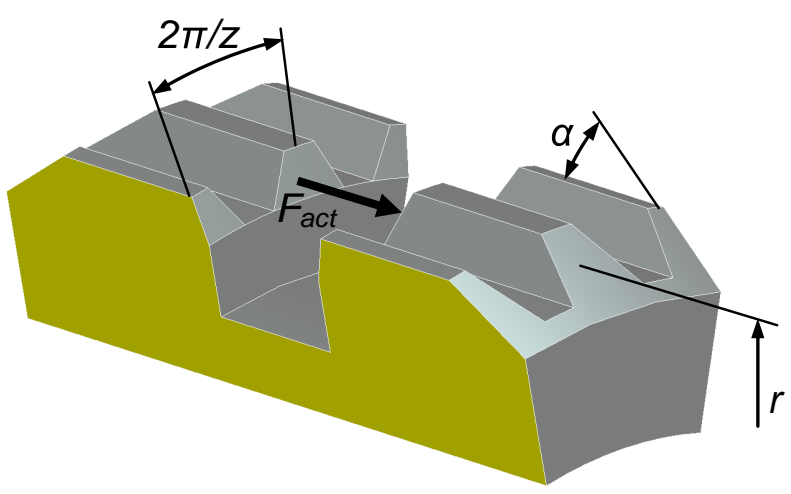

Fig. 3. Section of the sliding dog geometry

\subsection{Impact of tooth faces}

At the impact of the tooth faces of the gear wheel and the sliding dog, the axial velocity of the sliding dog rapidly decreases from $v_{0}$ to 0 as a result of a peak in the contact force. Let $F_{\text {imp }}(t)$ denote the axial component of the contact force (Fig. 4) during the impact $t \in\left[t_{0}, t_{1}\right]$. The $t_{1}-t_{0}$ time range of the impact and the $\Delta s_{i m p}$ axial displacement of the sliding during the impact are considered negligible. Assuming a tangential friction force, the angular accelerations and the change in the momen- 
tum of the sliding dog are:

$$
\begin{aligned}
\varepsilon_{i}^{i m p}(t) & =(-1)^{i} \operatorname{sgn}\left(\Delta \omega_{0}\right) \cdot \frac{\mu F_{i m p}(t)}{\sin \alpha} r \frac{1}{J_{i}}, t \in\left[t_{0}, t_{1}\right], \\
i & =1,2
\end{aligned}
$$

$$
\begin{aligned}
& m_{2} v_{0}=\int_{t_{0}}^{t_{1}}\left[F_{\text {imp }}(t)-F_{a c t}\right] d t= \\
& \int_{t_{0}}^{t_{1}} F_{i m p}(t) d t-F_{a c t}\left(t_{1}-t_{0}\right) \approx \int_{t_{0}}^{t_{1}} F_{i m p}(t) d t
\end{aligned}
$$

Using (2) and (3), the changes in mismatch speed and relative position during the impact are:

$$
\begin{gathered}
\Delta \omega_{i m p}=\int_{t_{0}}^{t_{1}}\left[\varepsilon_{1}^{i m p}(t)-\varepsilon_{2}^{i m p}(t)\right] d t= \\
\quad-s g n\left(\Delta \omega_{0}\right) \frac{\mu r m_{2} v_{0}}{\sin \alpha} \frac{J_{1}+J_{2}}{J_{1} J_{2}} \\
\Delta \varphi_{i m p}=\int_{t_{0}}^{t_{1}}\left[\omega_{1}(t)-\omega_{2}(t)\right] d t=\Delta \omega_{0}\left(t_{1}-t_{0}\right)- \\
\frac{\mu r m_{2}}{\sin \alpha} \frac{J_{1}+J_{2}}{J_{1} J_{2}}\left[v_{0}\left(t_{1}-t_{0}\right)-\Delta s_{i m p}\right] \approx 0
\end{gathered}
$$

Though the change in the relative position during the impact is negligible, the change in the mismatch speed is not. If $\left|\Delta \omega_{0}\right| \leq$ $\left|\Delta \omega_{i m p}\right|$, the mismatch speed of the dogs becomes zero during the impact:

$$
\begin{gathered}
\Delta \omega_{1}=\omega_{1}\left(t_{1}\right)-\omega_{2}\left(t_{1}\right)= \\
\operatorname{sgn}\left(\Delta \omega_{0}\right) \cdot \max \left(\left|\Delta \omega_{0}\right|-\left|\Delta \omega_{i m p}\right|, 0\right)
\end{gathered}
$$

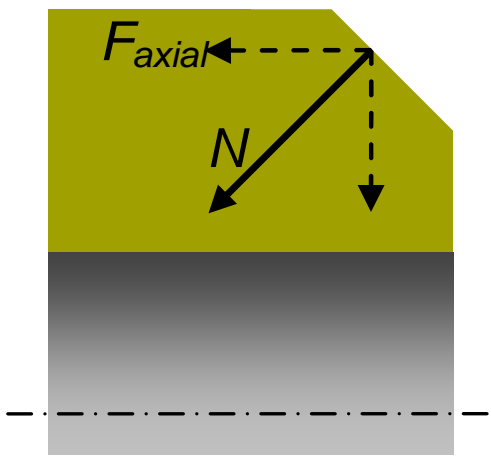

Fig. 4. Contact force on the sliding dog
(4) Using (6), (7), (8) and (9), the change in the relative position is obtained as:

$$
\begin{aligned}
& \Delta \varphi_{f f}=\int_{t_{1}}^{t_{1}+t_{f f}}\left[\omega_{1}(t)-\omega_{2}(t)\right] d t= \\
& \frac{\left[\max \left(\left|\Delta \omega_{0}\right|-\left|\Delta \omega_{i m p}\right|, 0\right)\right]^{2}}{\frac{2 \mu r F_{a c t}}{\sin \alpha} \frac{J_{1}+J_{2}}{J_{1} J_{2}}}
\end{aligned}
$$

\section{.3 Face friction phase}

Since the sliding dog does not move axially when the faces are in contact, the axial component of the contact force (Fig. 4) is of the same size as the actuator force. The angular accelerations caused by the face friction torque and the angular velocities are as follows:

$$
\begin{gathered}
\varepsilon_{i}=(-1)^{i} \operatorname{sgn}\left(\Delta \omega_{0}\right) \cdot \frac{\mu r F_{a c t}}{\sin \alpha} \frac{1}{J_{i}}, t>t_{1}, i=1,2 \\
\omega_{i}(t)=\omega_{i}\left(t_{1}\right)+\int_{t_{1}}^{t} \varepsilon_{i}(\tau) d \tau, t>t_{1}, i=1,2
\end{gathered}
$$
tion torque reduces the mismatch speed to zero and let $t_{f f}$ denote the time need for that. Equating (8) for $i=1$ and $i=2$ and considering (6) and (7) gives:

$$
t_{f f}=\frac{\max \left(\left|\Delta \omega_{0}\right|-\left|\Delta \omega_{i m p}\right|, 0\right)}{\frac{\mu r F_{a c t}}{\sin \alpha} \frac{J_{1}+J_{2}}{J_{1} J_{2}}}
$$

\section{Probability of the successful engagement}

Let $\Delta \varphi_{e}$ denote the relative angular displacement needed for the teeth of the sliding dog to become able to enter the slots of the gear wheel (Fig. 5). The engagement is successful if and only if

$$
\Delta \varphi_{e} \leq \Delta \varphi_{f f}
$$

$\Delta \varphi_{e}$ depends on the $\xi$ initial relative angular position of the sliding dog and the gear wheel that changes from one gear shift to another. Since $\xi$ is unknown but periodic by the pitch $2 \pi \cdot z^{-1}$, it is considered to be a random variable of uniform distribution [5] on the interval

$$
\xi \in\left[0 ; \frac{2 \pi}{z}\right]
$$

The definition of $\xi$ is shown in Fig. 5 as the angular distance between the marked tooth sides. The faces are overlapping if $0 \leq \xi<2 \pi z^{-1}-\Phi_{b}$, and the sliding dog is free to enter the slots of the gear wheel if $2 \pi z^{-1}-\Phi_{b} \leq \xi \leq 2 \pi z^{-1} . \Delta \varphi_{e}$ can be given as a function of $\xi$ :

$$
\Delta \varphi_{e}(\xi)=\left\{\begin{array}{c}
\frac{2 \pi}{z}-\Phi_{b}-\xi, \text { if } 0 \leq \xi<\frac{2 \pi}{z}-\Phi_{b} \\
0, \text { if } \frac{2 \pi}{z}-\Phi_{b} \leq \xi<\frac{2 \pi}{z}
\end{array}\right.
$$

Let us assume that the face contact is present until the face fric- 


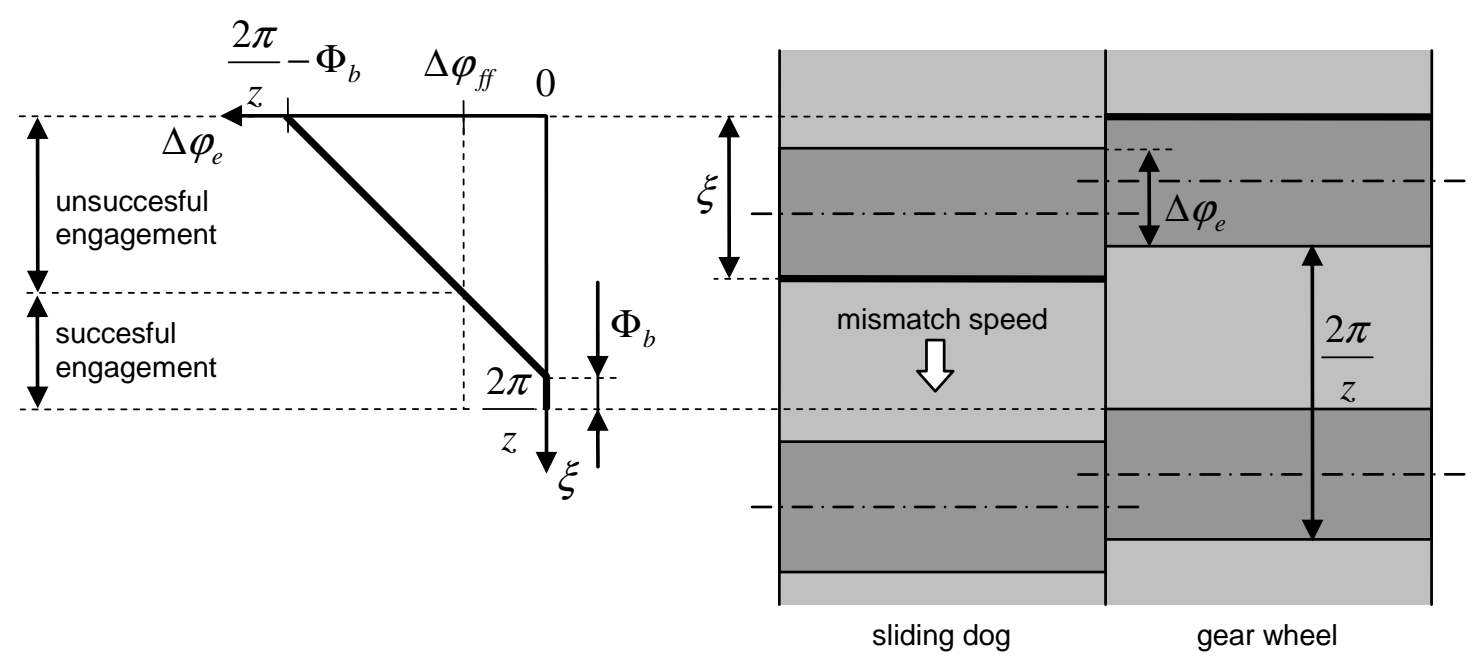

Fig. 5. Relative initial position and the geometrical probability of the successful engagement

Let $p$ denote the probability of the success of the engagement. Using (11) and (13) we obtain:

$$
\begin{array}{r}
p=P\left(\Delta \varphi_{e} \leq \Delta \varphi_{f f}\right)= \\
\left\{\begin{array}{l}
1, \text { if } \Delta \varphi_{f f}>\frac{2 \pi}{z}-\Phi_{b} \\
\frac{z\left(\Delta \varphi_{f f}+\Phi_{b}\right)}{2 \pi}, \text { if } \Delta \varphi_{f f} \leq \frac{2 \pi}{z}-\Phi_{b}
\end{array}\right.
\end{array}
$$

Considering the backlash angle $\Phi_{b}$, the teeth of the sliding dog enter the slots of the gear wheel without any contact of the faces with a geometrical probability of

$$
p_{0}=\frac{z \cdot \Phi_{b}}{2 \pi}
$$

Finally, combining (10), (14) and (15) gives the probability of the successful engagement as a function of the initial mismatch speed:

$$
\left\{\begin{aligned}
p_{0}, \text { if }\left|\Delta \omega_{0}\right|-\left|\Delta \omega_{i m p}\right| & \leq 0 \\
\frac{z\left(\left|\Delta \omega_{0}\right|-\left|\Delta \omega_{i m p}\right|\right)^{2}}{\frac{4 \pi \mu r F_{a c t}}{\sin \alpha} \frac{J_{1}+J_{2}}{J_{1} J_{2}}} & +p_{0}, \text { if } 0<\left|\Delta \omega_{0}\right|-\left|\Delta \omega_{i m p}\right| \\
& \leq \sqrt{2\left(\frac{2 \pi}{z}-\Phi_{b}\right) \frac{\mu r F_{a c t}}{\sin \alpha} \frac{J_{1}+J_{2}}{J_{1} J_{2}}} \\
1, \text { if }\left|\Delta \omega_{0}\right|-\left|\Delta \omega_{i m p}\right| & >\sqrt{2\left(\frac{2 \pi}{z}-\Phi_{b}\right) \frac{\mu r F_{a c t}}{\sin \alpha} \frac{J_{1}+J_{2}}{J_{1} J_{2}}}
\end{aligned}\right.
$$

Referring to a heavy duty commercial vehicle equipped with a 12-speed AMT with driveline, dog geometry and actuator parameters matched to the parameters of the test bench used for validation in Chapter 6, Fig. 6 shows the probability of the success of the engagement as a function of the initial mismatch speed of the dog clutch involved in the gear shift for gears 1, 3 and 5. Since the probability functions depend on the reduced inertias of the driveline and thus on the gearbox ratios, they vary from gear to gear even at a given gearbox and dog geometry. Note that though $p_{1}(3.6 \mathrm{rad} / \mathrm{s})=1$ for the $1^{\text {st }}$ gear, engaging the $5^{\text {th }}$ gear with the same mismatch speed gives only $p_{5}(3.6 \mathrm{rad} / \mathrm{s})=0.56$.

\section{The optimal mismatch speed}

Considering (16) and (4), the lowest positive mismatch speed with $p=1$ can be written as

$\Delta \omega_{o p t}=\frac{\mu r m_{2} v_{0}}{\sin \alpha} \frac{J_{1}+J_{2}}{J_{1} J_{2}}+\sqrt{2\left(\frac{2 \pi}{z}-\Phi_{b}\right) \frac{\mu r F_{a c t}}{\sin \alpha} \frac{J_{1}+J_{2}}{J_{1} J_{2}}}$

Since $\Delta \omega_{o p t}$ and $-\Delta \omega_{\text {opt }}$ are the lowest mismatch speeds ensuring the success of the engagement, they can be considered as optimal and should be preferred at gear shifts. Note that the optimal mismatch speed is determined by parameters not only referring to the dog geometry, but to the driveline and the actuator as well and also varies from gear to gear.

Most of the parameters in (17) can only be influenced at the design stage, except for the gearbox actuation parameters. Reducing the impact speed and limiting the actuator force during the face contact is favorable to achieve smooth gear shifts. This latter case is just the opposite as desired for the synchromesh where a high synchronizing force is needed.

\section{The mismatch speed at the engagement and the torque peak of the vibrations}

Since $\Delta \varphi_{e}(\xi)$ is a random variable, so is $\Delta \omega_{2}$ at a given $\Delta \omega_{0}$. Using (7) and (13) we obtain:

$$
\begin{aligned}
& \left|\Delta \omega_{2}(\xi)\right|= \\
& \left\{\begin{array}{l}
\left|\Delta \omega_{0}\right|, \text { if } \frac{2 \pi}{z}-\Phi_{b} \leq \xi<\frac{2 \pi}{z} \\
\left|\Delta \omega_{0}\right|-\left|\Delta \omega_{i m p}\right|-\sqrt{2\left(\frac{2 \pi}{z}-\Phi_{b}-\xi\right) \frac{\mu r F_{a c t}}{\sin \alpha} \frac{J_{1}+J_{2}}{J_{1} J_{2}}} \\
\text { if } \frac{2 \pi}{z}-\Phi_{b}-\Delta \varphi_{f f} \leq \xi<\frac{2 \pi}{z}-\Phi_{b}
\end{array}\right.
\end{aligned}
$$

where cases for engagement without and with face contact are separated. There is no engagement when

$0 \leq \xi<\frac{2 \pi}{z}-\Phi_{b}-\Delta \varphi_{f f}$, so that case is not considered in (18). 
Fig. 6. Probability of the successful engagement for gears 1,3 and 5

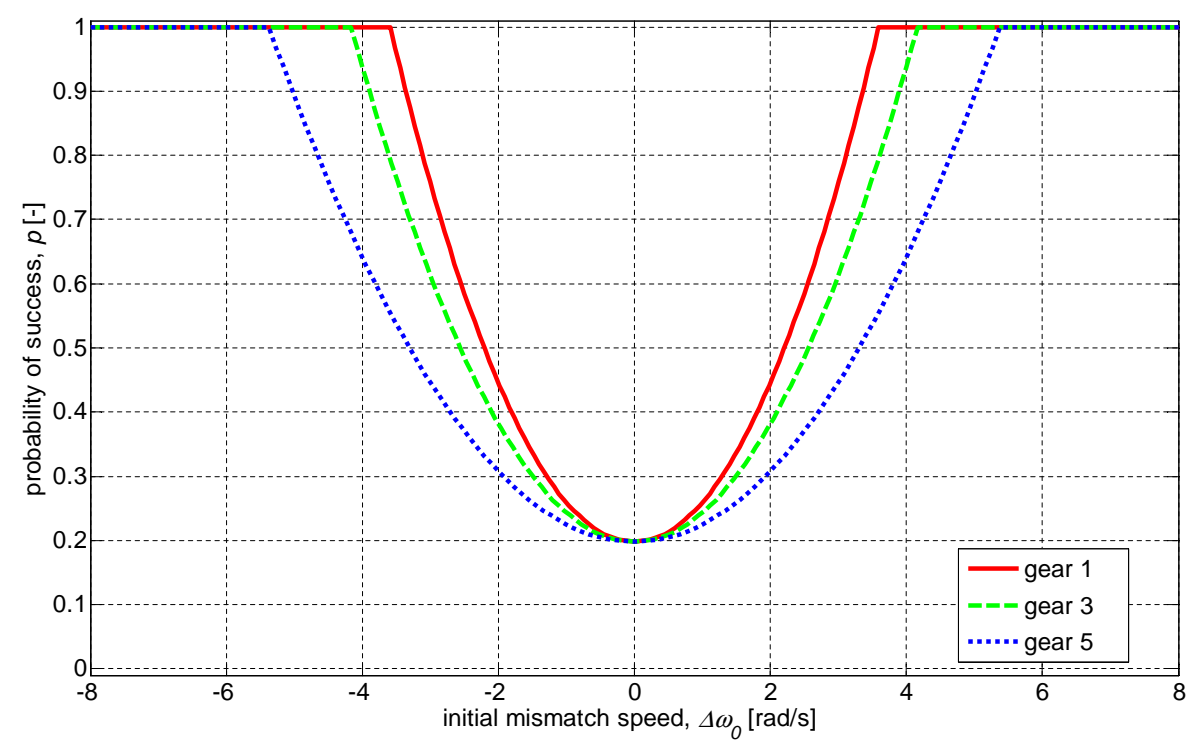

Fig. 7] shows the mismatch speed at the engagement as a function of $\xi$ for three different initial mismatch speeds: for the optimal, for a lower and for a higher one. Calculation is done for the $1^{s t}$ gear with the same parameters as used in Fig. 6

According to (1), $T_{\max }$ is also a random variable, with the same distribution as $\left|\Delta \omega_{2}\right|$. Calculating (1) with the mismatch speed according to (17) and neglecting the speed loss (4) caused by the impact, we obtain:

$$
\left.T_{\max }\right|_{\Delta \omega_{2}=\Delta \omega_{o p t} \wedge \frac{2 \pi}{z}-\Phi_{b} \leq \xi \leq \frac{2 \pi}{z}} \approx \sqrt{2 k\left(\frac{2 \pi}{z}-\Phi_{b}\right) \frac{\mu F_{a c t}}{\sin \alpha} r}
$$

(19) is an estimation for the maximal possible value of the torque peak assuming that the initial mismatch speed is $\Delta \omega_{\text {opt }}$. Note that (19) does not contain driveline parameters any more, only parameters of the dog clutch and the actuation.

\section{Measurement results}

To verify the theoretical results for the probability curves (16), measurements were made on a test bench equipped with a heavy duty AMT. Gears 1, 3 and 5 were shifted as any shift between those gears and the neutral requires only the shifting of one of the dog clutches of the gearbox. The countershaft brake was not used since the brake torque was not considered in the theoretical part, either. The actual mismatch speed of the dogs was calculated using the signals of the built-in speed sensors of the gearbox mounted on the input and output shafts.

Several shifting attempts were completed according to an automatic sequence. First the input shaft speed was increased then driving torque was reduced to zero. The input- and countershafts subsequently began to slow down because of the friction losses. The gear was shifted automatically when a pre-defined mismatch speed was reached. The mismatch speed $\Delta \omega_{0}$ at the face contact position of the sliding dog and the outcome of the attempt were registered. Only non-negative mismatch speeds were considered.
Mismatch speed intervals for $\Delta \omega_{0}$ were defined for all the three gears. Tab. 1 contains the interval limits, the total number of trials $(n)$, the number of successful trials $(k)$ and the relative frequency of the success $(k / n)$ for all intervals. Statistical evaluation is based on a total number of 5072 gear shifts.

According to the law of large numbers [5], the relative frequency and the probability of the success of the engagement are not independent:

$$
P\left(\left|\frac{k}{n}-p\right| \geq \varepsilon\right) \leq \frac{p(1-p)}{\varepsilon^{2} n}=\frac{1-4(p-0.5)^{2}}{4 \varepsilon^{2} n} \leq \frac{1}{4 \varepsilon^{2} n}
$$

Using $\varepsilon=\sqrt{4 \delta n}$ we obtain that

$$
p \in\left[\max \left(0, \frac{k}{n}-\sqrt{\frac{1}{4 \delta n}}\right), \min \left(1, \frac{k}{n}+\sqrt{\frac{1}{4 \delta n}}\right)\right]
$$

is fulfilled with a probability of at least $1-\delta$. In the following we use $\delta=0.1$, so the confidence intervals are calculated for the probability of 0.9 .

Class limits in Table tab1 were determined to have a sufficient number of trials in each interval to ensure confidence intervals narrow enough.

Figures from Fig. 8 to 10 show the theoretical results for $\Delta \omega_{0} \geq 0$ and the confidence intervals obtained by the evaluation of the measurements.

Though the applied model is quite simple, the theoretical expression for the probability of the success of the engagement shows very good correlation with the measurement results.

According to the measurements, the variation of probability of the successful engagement with the initial mismatch speed and its dependence on the shifted gear fits the expectations.

\section{Summary and conclusions}

In this paper, the following results were achieved:

1 The mechanical model of a face dog clutch was built up with 
Fig. 7. Mismatch speed at the engagement as a function of the random initial position

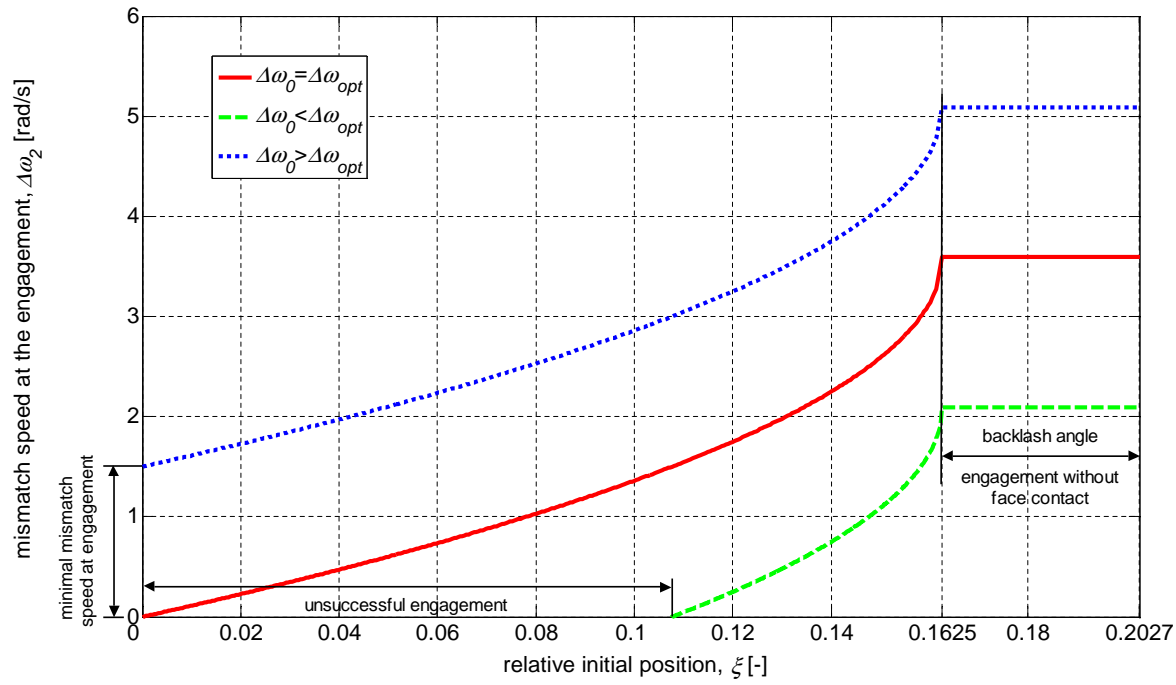

Tab. 1. Statistical evaluation of measurement results for gears 1,3 and 5

\begin{tabular}{|c|c|c|c|c|c|c|c|c|c|c|c|c|c|c|c|}
\hline & \multicolumn{5}{|c|}{ Gear 1} & \multicolumn{5}{|c|}{ Gear 3} & \multicolumn{5}{|c|}{ Gear 5} \\
\hline 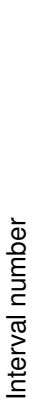 & 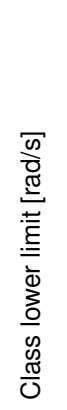 & 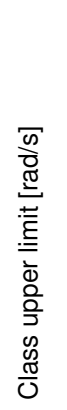 & 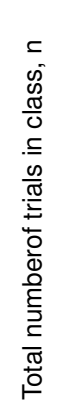 & 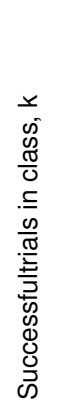 & 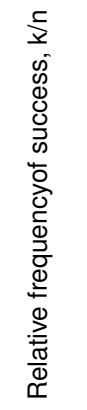 & 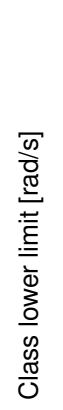 & 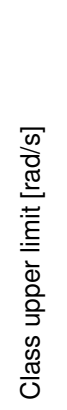 & 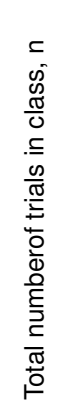 & 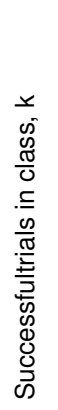 & 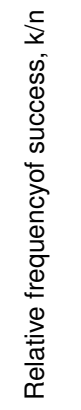 & 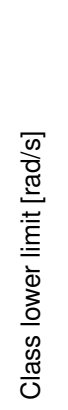 & 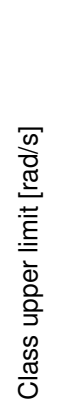 & 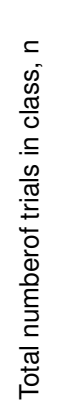 & 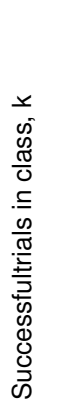 & 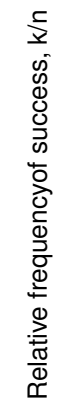 \\
\hline 1 & 0 & 0.01 & 255 & 49 & 0.192 & 0 & 0.01 & 241 & 49 & 0.203 & 0 & 0.01 & 252 & 53 & 0.210 \\
\hline 2 & 0.01 & 0.9 & 250 & 47 & 0.188 & 0.01 & 0.8 & 250 & 45 & 0.180 & 0.01 & 0.6 & 203 & 54 & 0.266 \\
\hline 3 & 0.9 & 1.8 & 187 & 51 & 0.273 & 0.8 & 1.7 & 240 & 54 & 0.225 & 0.6 & 1.3 & 205 & 56 & 0.273 \\
\hline 4 & 1.8 & 2.7 & 198 & 78 & 0.394 & 1.7 & 2.6 & 220 & 76 & 0.345 & 1.3 & 2.0 & 182 & 46 & 0.253 \\
\hline 5 & 2.7 & 3.6 & 173 & 113 & 0.653 & 2.6 & 3.5 & 208 & 102 & 0.490 & 2.0 & 3.0 & 231 & 91 & 0.394 \\
\hline 6 & 3.6 & 4.5 & 70 & 70 & 1.00 & 3.5 & 4.5 & 313 & 274 & 0.875 & 3.0 & 4.0 & 350 & 175 & 0.500 \\
\hline 7 & 4.5 & 5.4 & 71 & 71 & 1.00 & 4.5 & 5.4 & 123 & 122 & 0.992 & 4.0 & 5.0 & 291 & 252 & 0.866 \\
\hline 8 & 5.4 & 6.3 & 81 & 81 & 1.00 & 5.4 & 6.3 & 86 & 86 & 1.00 & 5.0 & 6.0 & 128 & 127 & 0.992 \\
\hline \multirow[t]{2}{*}{9} & 6.3 & 8.0 & 80 & 80 & 1.00 & 6.3 & 8.0 & 81 & 81 & 1.00 & 6.0 & 8.0 & 103 & 103 & 1.00 \\
\hline & \multicolumn{2}{|c|}{ Total: } & 1365 & 640 & & \multicolumn{2}{|c|}{ Total: } & 1762 & 889 & & \multicolumn{2}{|c|}{ Total: } & 1945 & 957 & \\
\hline
\end{tabular}

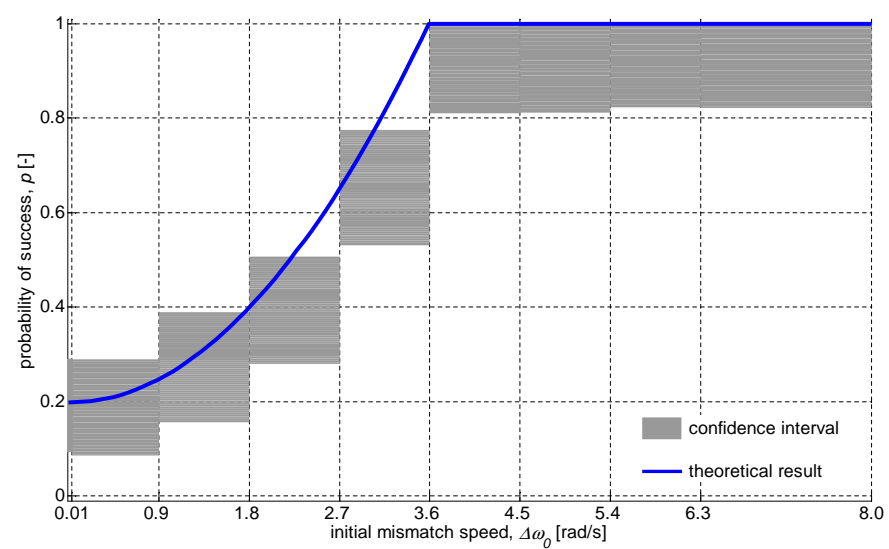

Fig. 8. Measurement and theoretical results for the probability of the successful engagement for gear 1

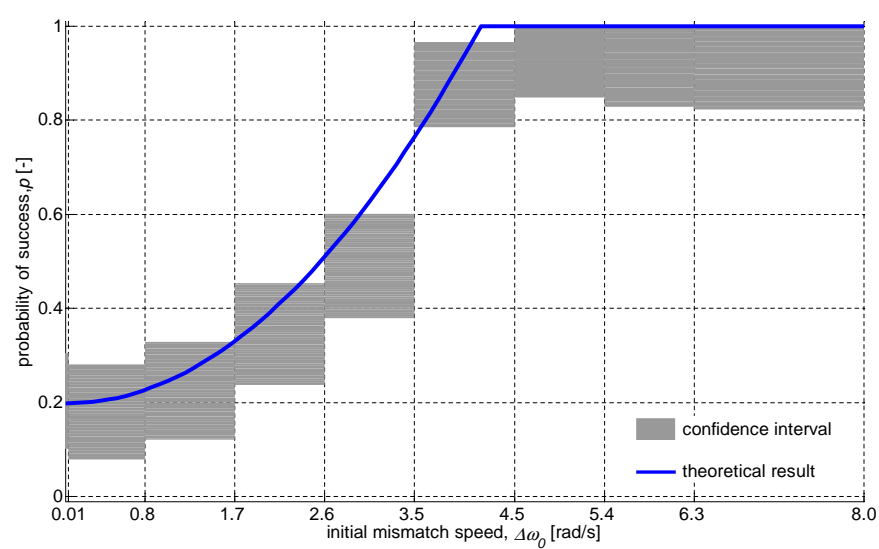

Fig. 9. Measurement and theoretical results for the probability of the successful engagement for gear 3 


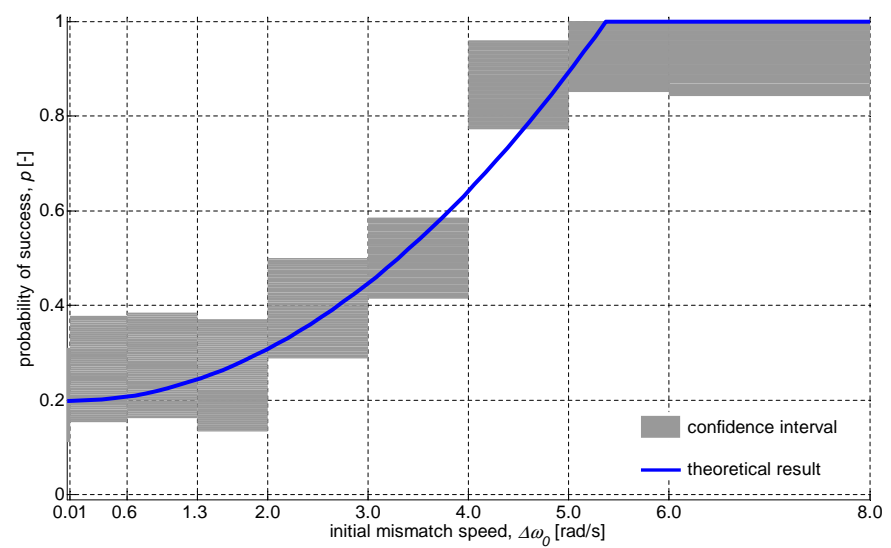

Fig. 10. Measurement and theoretical results for the probability of the successful engagement for gear 5

driveline inertias reduced to the gear wheel and to the sliding dog.

2 The non-deterministic outcome of the engagement process was described, giving the probability of the successful engagement in an analytical form. It was shown that the engagement probability curves depend on the shifted gear at a given dog geometry.

3 The uncertainty of the mismatch speed at the engagement and the torque peak of the post engagement torsional vibrations were described.

4 Theoretical results obtained for the engagement probability were validated through statistical evaluation of test bench measurement data for different gears of a heavy duty AMT and good correlation was found between the theoretical and measurement results.

Results of this paper can be used for the improvement of the gear shift comfort of any gearbox including face dog clutches.

\section{References}

1 ZF ASTronic Technical Manual for installation, function and initial setup.

2 Bóka G, Márialigeti J, Lovas L, Trencséni B, External synchronization strategies for automated mechanical transmissions with face dog clutch and countershaft brake, Papers of the $8^{\text {th }}$ International Multidisciplinary Conference, 2009.

3 Lovas L, Play D, Márialigeti J, Rigal J F, Mechanical behaviour simulation for synchromesh mechanism improvements, Proc. Instn Mech. Engrs, Part D: J Automobile Engineering, 2006.

4 Laird M P B, Lawton B, Gregory R P, Dog clutches for rapid gear changes in automotive gearboxes, Proceedings of the Institution of Mechanical Engineers, First International Conference, Gearbox Noise and Vibrations, IMechE, 1990, pp. 103-112.

5 Dougherty E R, Probability and statistics for the engineering, computing, and physical sciences, Prentice-Hall, Inc., Upper Saddle River, NJ, USA, 1990. 